\title{
Approximation of the Spectrum of an Operator Given by the Magnetohydrodynamic Stability of a Plasma
}

\author{
By Yves Jaccard and Hugo Evéquoz
}

\begin{abstract}
The study of the magnetohydrodynamic (MHD) stability of a plasma in a toroidal configuration leads to a problem of computing the spectrum of a noncompact selfadjoint operator $T$. The spectrum of $T$ will be approximated by the eigenvalues of $T_{h}$, a Galerkin approximation of $T$.

We present a two-dimensional model problem with two components containing most difficulties arising in the physical problem. We give subspaces and prove sufficient conditions for obtaining convergence using partial regularity of $T$.
\end{abstract}

1. Introduction. We consider an equilibrium plasma in a toroidal geometry $\Omega_{p}$. Linearizing the evolution equations near the equilibrium position characterized by the magnetic field $\vec{B}_{0}$, the pressure $p_{0}$ and the density $\rho_{0}$, the Eulerian equations can be written in the form

$$
\rho_{0}\left(\partial_{t}\right)^{2} \vec{\xi}=\vec{F}(\vec{\xi}),
$$

where $\vec{\xi}$ is the displacement in the variables of Lagrange and where

$$
\begin{aligned}
\vec{F}(\vec{\xi})= & \operatorname{gradd}\left(\vec{\xi} \cdot \operatorname{gradd} p_{0}+\gamma p_{0} \operatorname{div} \vec{\xi}\right) \\
& +\frac{1}{4 \Pi}\left(\operatorname{rot} \vec{B}_{0} \wedge \vec{Q}+\operatorname{rot} \vec{Q} \wedge \vec{B}_{0}\right)
\end{aligned}
$$

with $\vec{Q}=\operatorname{rot}\left(\vec{\xi} \wedge \vec{B}_{0}\right) ;$ see [7, p. 252].

In order to study the stability of this equilibrium, we search solutions of (1.1) of the form

$$
\vec{\xi}(x, t)=\vec{\xi}(x) e^{i \omega t} .
$$

Using integration by parts, the potential energy

$$
W_{p}(\vec{\xi})=-\frac{1}{2} \int_{\Omega_{p}} \vec{\xi} \cdot \vec{F}(\vec{\xi}) d x
$$

can be written in the following symmetric form

$$
\begin{array}{r}
W_{p}(\vec{\xi})=\frac{1}{2} \int_{\Omega_{p}}\left\{\left|\overrightarrow{\operatorname{rot}}\left(\vec{\xi} \wedge \vec{B}_{0}\right)+\left(\overrightarrow{n_{0}} \cdot \vec{\xi}\right)\left(\overrightarrow{j_{0}} \wedge \vec{n}_{0}\right)\right|^{2}+\gamma p_{0}(\operatorname{div} \vec{\xi})^{2}\right. \\
\left.-\left(\vec{n}_{0} \cdot \vec{\xi}\right)\left(\vec{j}_{0} \wedge \vec{n}_{0}\right)\left(\overrightarrow{B_{0}} \cdot \vec{\nabla}\right) \vec{n}_{0}\right\} d x,
\end{array}
$$

Received July 15, 1980; revised January 13, 1982.

1980 Mathematics Subject Classification. Primary 65N25, 76W05. 
where $\overrightarrow{j_{0}}=\operatorname{rot} \vec{B}_{0} / 4 \Pi$ and $\vec{n}_{0}=-\left|\operatorname{gra} d p_{0}\right|^{-1}$ gräd $p_{0}$ is the external unit normal to the isobar.

Moreover, by the energy principle, $\vec{\xi}(x)$ is a stationary point of the form

$$
W_{p}(\vec{\xi})-W_{c}(\vec{\xi})
$$

where

$$
W_{c}(\vec{\xi})=\frac{1}{2} \omega^{2} \int_{\Omega_{p}} \rho_{0}|\vec{\xi}|^{2} d x
$$

is the kinetic energy.

Then the solutions (1.3) belong to the invariant subspaces of the selfadjoint operator $T$ defined by

$$
a(T \vec{\xi}, \vec{\zeta})=b(\vec{\xi}, \vec{\zeta})
$$

for all displacement $\vec{\zeta}$, where $a(\cdot, \cdot)$ and $b(\cdot, \cdot)$ are sesquilinear forms such that $a(\vec{\xi}, \vec{\xi})=W_{p}(\vec{\xi})$ and $b(\vec{\xi}, \vec{\xi})=W_{c}(\vec{\xi}) / \omega^{2}$

On the other hand, $1 / \omega^{2}$ belongs to the spectrum of $T$ and the stability criterion bears upon the sign of $\omega^{2}$.

Supposing the revolution symmetry of the equilibrium system relative to the axis $O_{z}$. Parametrizing the toroidal domain $\Omega_{p}$ by the cylindrical coordinates $(r, \varphi, z)$, eliminating the angular variable $\varphi$ by means of Fourier series and by a change of the two other independent variables in some polar-like coordinates using physical properties of $\vec{B}_{0}, p_{0}$ and $\rho_{0}$, we describe a section of the torus with specific variables $(s, \chi)$ lying in the rectangular domain $\Omega=[0,1] \times[0,2 \Pi]$. The magnetic surfaces are given by the equation $s=$ constant.

Applying simultaneously a change of the dependent variables $\left(\xi_{r}, \xi_{\varphi}, \xi_{z}\right) \rightarrow$ $(X, Y, V), W_{p}(\vec{\xi})$ and $W_{c}(\vec{\xi})$ can be written

$$
\begin{aligned}
W_{p}=\frac{\Pi}{2} \int_{\Omega} \frac{d s}{s} d \chi & \left\{\frac{4 \psi_{s} \tau^{3} s^{2}}{q r^{4} B_{p}^{2}}\left|\frac{1}{q} \partial_{\chi} X+i n X\right|^{2}+\frac{\tau^{3}}{q \psi_{s}}\left|\partial_{s} X+\partial_{\chi} V\right|^{2}\right. \\
& +\frac{q r^{2} B_{p}^{2}}{\tau \psi_{s} s^{2}} \mid s \partial_{s}\left(\frac{\tau}{q} X\right)+s \partial_{\chi}\left(\frac{\tau}{q} V\right)+s \theta \tau\left(\frac{1}{q} \partial_{\chi} X+i n X\right)
\end{aligned}
$$

$$
\begin{array}{r}
-s \tau\left(\frac{1}{q} \partial_{\chi} V+i n V\right)+\left.\frac{2 \psi_{s} j_{\varphi} \tau s^{2}}{r q B_{p}^{2}} X\right|^{2} \\
+\frac{\gamma p_{0} \tau}{q r^{2} \psi_{s}}\left|\partial_{s}\left(r^{2} X\right)+\partial_{\chi}\left(r^{2} V\right)+q\left(\frac{1}{q} \partial_{\chi}\left(r^{2} Y\right)+i n r^{2} Y\right)\right|^{2} \\
\left.-\frac{8 \tau K r^{2} \psi_{s} s^{2}}{q}|X|^{2}\right\},
\end{array}
$$

$$
W_{c}=\omega^{2} \Pi \int_{\Omega} \frac{d s}{s} d \chi \rho_{0}\left\{\frac{2 \psi_{s} \tau s^{2}}{q B_{p}^{2}}|X|^{2}+\frac{r^{4} q \tau}{2 \psi_{s}}|Y|^{2}+\frac{r^{6} q B_{p}^{2}}{2 \tau \psi_{2}}|V+Y-\theta X|^{2}\right\}
$$


The quantities $\psi_{s}, \tau, q, B_{p}, \theta, j_{\varphi}$, and $K$ depend on the geometry $\Omega_{p}$ and on the fields $\vec{B}_{0}$ and $p_{0}$; see [1] and [5].

In order to eliminate the singularity of (1.9) and (1.10) for $s=0$, we consider the operator $T$ associated to a plasma without vacuum region evolving in a toroidal crown (let us mention that this singularity is such that the space of displacements is not a product space; see [6, Annexe A]).

The spectral convergence of a Galerkin discretization of $T$ for a one-dimensional plasma situation has been studied by J. Rappaz [8]. The two-dimensional problem involves new types of difficulties and requires other methods of treatment. In particular, $V$ and $Y$ admit in (1.9) only one partial derivative with respect to $\chi$, and this fact requires the construction of an unconventional Sobolev space. On the other hand, an untraditional result of directional and global regularity of the operator allows for coupling terms of (1.9).

For the sake of simplicity, we shall however restrict ourselves here to a similar two-component problem. In our thesis, we have obtained results for a rather general framework which includes in particular the one-dimensional plasma situation studied by J. Rappaz in [8]. On the other hand, H. Evequoz analyzes in [3] a nonconforming finite element method for the same type of problem. These numerical schemes are used successfully in several laboratories for plasma physics, in particular in the CRPP of the Federal Institute of Technology, Lausanne.

Let us describe a theoretical general method for spectral approximation of $T$ (Galerkin's method): Let $U$ be a complex Hilbert space with scalar product $(\cdot, \cdot)_{U}$ and $\left\{U_{h}\right\}_{0<h<1}$ be a family of closed subspaces. Let $T: U \rightarrow U$ and $T_{h}: U_{h} \rightarrow U_{h}$ be the operators defined by $a(T u, v)=b(u, v) \forall u, v \in U$ and $a\left(T_{h} u, v\right)=b(u, v)$ $\forall u, v \in U_{h}$, where $a(\cdot, \cdot)$ and $b(\cdot, \cdot)$ are continuous sesquilinear forms (linear in the first argument and antilinear in the second). We suppose that $a(\cdot, \cdot)$ is coercive on $U$. Let $p_{h}: U \rightarrow U_{h}$ be the $(\cdot, \cdot)_{U}$ projection.

J. Descloux, N. Nassif and J. Rappaz showed in [2] that the eigenvalues and eigenvectors of $T_{h}$ are "good approximations" of the spectrum and invariant subspaces of $T$ if the following properties hold:

$$
\begin{gathered}
\lim _{h \rightarrow 0} \sup _{u \in U_{h} ;\|u\|_{U}=1}\left\|\left(I-p_{h}\right) T u\right\|_{U}=0 . \\
\lim _{h \rightarrow 0}\left\|\left(I-p_{h}\right) u\right\|_{U}=0, \quad \forall u \in U .
\end{gathered}
$$

When $T$ is compact, the following classical proposition shows that (P2) implies (P1).

Proposition 1.1. Let $B$ be a Banach space with norm $\|\cdot\|_{B}$ and $Z: B \rightarrow U$ a linear continuous operator. If property ( $\mathrm{P} 2)$ holds and if $Z$ is compact, then we have

$$
\lim _{h \rightarrow 0} \sup _{u \in B ;\|u\|_{U}=1}\left\|\left(I-p_{h}\right) Z u\right\|_{U}=0 .
$$

In this paper, we shall construct a family of finite element subspaces $\left\{U_{h}\right\}_{0<h<1}$ associated to the preceding MHD stability problem for which we shall verify that conditions (P1) and (P2) are satisfied.

2. The Exact Problem. Partial Regularity of the Operator. Let $\Omega=(0,1) \times(0,1)$ $\subset \mathbf{R}^{2}$. We denote by $(x, y)$ the independent variables in $\mathbf{R}^{2}$.

Let $\mathscr{D}=\left\{u / \bar{\Omega} \mid u \in C^{\infty}(\mathbf{R} \times[0,1]), u\right.$ is 1-periodic in the variable $\left.x\right\} \subset C^{\infty}(\bar{\Omega})$ and $\mathscr{D}_{0}=\{u \in \mathscr{D} \mid \exists \varepsilon>0$ depending on $u$ such that $\operatorname{supp}(u) \subset[0,1] \times[\varepsilon, 1-\varepsilon]\}$. 
Let $V^{1}$ be the completion of $\mathscr{D}$ for the norm

$$
\|u\|_{1, \Omega}=\left(\|u\|_{0, \Omega}^{2}+\left\|\partial_{x} u\right\|_{0, \Omega}^{2}+\left\|\partial_{y} u\right\|_{0, \Omega}^{2}\right)^{1 / 2}
$$

$\left(\|\cdot\|_{0, \Omega}\right.$ is the norm in $L^{2}(\Omega)$ ), $U^{1}$ be the closure of $\mathscr{D}_{0}$ in $V^{1}, V^{2}$ be the completion of $\mathscr{D}$ for the norm $\|u\|_{1, x, \Omega}=\left(\|u\|_{0, \Omega}^{2}+\left\|\partial_{x} u\right\|_{0, \Omega}^{2}\right)^{1 / 2}, U^{2}$ be the closure of $\mathscr{D}_{0}$ in $V^{2}$ (we have $U^{2}=V^{2}$ ), $V=V^{1} \times V^{2}$ with the norm

$$
\|u\|_{V}=\left(\left\|u^{1}\right\|_{1, \Omega}^{2}+\left\|u^{2}\right\|_{1, x, \Omega}\right)^{1 / 2},
$$

where $u=\left(u^{1}, u^{2}\right) \in V$ and $U=U^{1} \times U^{2}$. We denote by $(\cdot, \cdot)_{V}$ the scalar product of $V$ which induces the norm $\|\cdot\|_{V}$.

Consider the hermitian sesquilinear forms $a(\cdot, \cdot)$ and $b(\cdot, \cdot): V \times V \rightarrow \mathbf{C}$ defined by $a(u, v)=\int_{\Omega}[v]^{*} A[u]$ and $b(u, v)=\int_{\Omega}\{v\}^{*} B\{u\}$ where $A$ (resp. $B$ ) is a $5 \times 5$ (resp. $2 \times 2)$ hermitian matrix with coefficients in $\mathscr{D},[u]={ }^{t}\left(u^{1}, \partial_{x} u^{1}, \partial_{y} u^{1}, u^{2}, \partial_{x} u^{2}\right)$, $[v]={ }^{t}\left(v^{1}, \partial_{x} v^{1}, \partial_{y} v^{1}, v^{2}, \partial_{x} v^{2}\right),\{u\}={ }^{t}\left(u^{1}, u^{2}\right)$ and $\{v\}={ }^{t}\left(v^{1}, v^{2}\right)$. Suppose that $a(\cdot, \cdot)$ is coercive on $U$, and define the linear continuous selfadjoint operator $T: U \rightarrow U$ by $a(T u, v)=b(u, v) \forall u, v \in U$.

THEOREM 2.1. If $u \in U$, then $\left(\partial_{x}\right)^{i} T u \in U \forall 0 \leqslant i \leqslant 2$, and there exists $c>0$ independent of $u$ such that

$$
\sum_{i=0}^{2}\left\|\left(\partial_{x}\right)^{i} T u\right\|_{V} \leqslant c\|u\|_{V} .
$$

The proof which relies strongly on the property of periodicity of $U$ is obtained in [6] by the differential quotient method; see, for example, [4].

3. Discretization Subspaces. The choice of the subspaces is connected to the coupling terms. Using the same subspaces for each component of the one-dimensional problem, Rappaz shows, for example in [8], the existence of a suite of eigenvalues of $T_{h}$ that converges in the resolvent set of $T$. This type of phenomenon, so-called "spectral pollution", can appear when property (P1) is unsatisfied. The intuitive argument that suggests the choice of the discretization subspaces is the following:

Let $\delta a(\cdot, \cdot)$ be the part of $a(\cdot, \cdot)$ exclusively constituted by coupling terms. Setting $a_{0}(\cdot, \cdot)=a(\cdot, \cdot)-\delta a(\cdot, \cdot)$, we define $T_{0}$ and $R$ by $a_{0}\left(T_{0} u, v\right)=b(u, v)$ and $a_{0}(R u, v)=\delta a(u, v)$. We have the relation

$$
(I+R)\left(T-T_{h}\right)=T_{0}-T_{0 h}-\left(R-R_{h}\right) T_{h} .
$$

Supposing (P1) for $T_{0}$, we must prove a similar property for $R$. For the term $\int_{\Omega} \partial_{y} u^{1} \bar{v}^{2}$, the approximation property holds when $\partial_{y} u^{1}$ belongs to the subspace discretizing the second component. In this way, we choose a subspace $U_{h}=U_{h}^{1} \times U_{h}^{2}$ such that $\partial_{y} u^{1}$ and $u^{2}$ have the same form, $\forall u=\left(u^{1}, u^{2}\right) \in U_{h}$.

Let $H^{1}(0,1)$ be the Sobolev space of the functions $f \in L^{2}(0,1)$ such that $f^{\prime} \in$ $L^{2}(0,1), H_{\#}^{1}(0,1)=\left\{f \in H^{1}(0,1) \mid f(0)=f(1)\right\}$ and $H_{0}^{1}(0,1)=\left\{f \in H^{1}(0,1) \mid f(0)\right.$ $=f(1)=0\}$.

For $r_{1}, r_{2} \in \mathbf{N}$, we denote by $\mathbf{P}_{r_{1}, r_{2}}$ the space of polynomials of two variables of degree $\leqslant r_{1}$ in the first variable and $\leqslant r_{2}$ in the second one. $\mathbf{P}_{r_{1}}$ is the space of polynomials of one variable of degree $\leqslant r_{1}$. 
For $0<h \leqslant 1$ such that $N_{h}=1 / h \in \mathbf{N}$, let $x_{i}=i h$ and $y_{j}=j h \forall 0 \leqslant i, j \leqslant N_{h}$, $R_{i j}=\left(x_{i-1}, x_{i}\right) \times\left(y_{j-1}, y_{j}\right) \forall 1 \leqslant i, j \leqslant N_{h}$ and $\mathscr{R}_{h}=\left\{R_{i j} \mid 1 \leqslant i, j \leqslant N_{h}\right\}$. Let $r \in \mathbf{N}, r \geqslant 1$ be fixed. Define

$$
\begin{aligned}
& U_{h}^{1}=\left\{u \in U^{1} \mid u / R \in \mathbf{P}_{r, r} \forall R \in \Re_{h}\right\}, \\
& U_{h}^{2}=\left\{u \in U^{2} \mid u / R \in \mathbf{P}_{r, r-1} \forall R \in \Re_{h}\right\},
\end{aligned}
$$

and $U_{h}=U_{h}^{1} \times U_{h}^{2}$. Note that $U_{h}^{2}$ contains discontinuous functions if $N_{h} \geqslant 2$. Set

$$
\begin{aligned}
P_{h}(0,1) & =\left\{f \in H_{\#}^{1}(0,1) \mid f /\left(x_{i-1}, x_{i}\right) \in \mathbf{P}_{r} \forall 1 \leqslant i \leqslant N_{h}\right\}, \\
Q_{h}^{1}(0,1) & =\left\{f \in H_{0}^{1}(0,1) \mid f /\left(y_{j-1}, y_{j}\right) \in \mathbf{P}_{r} \forall 1 \leqslant j \leqslant N_{h}\right\}, \\
Q_{h}^{2}(0,1) & =\left\{f \in L^{2}(0,1) \mid f /\left(y_{j-1}, y_{j}\right) \in \mathbf{P}_{r-1} \forall 1 \leqslant j \leqslant N_{h}\right\} .
\end{aligned}
$$

One can verify that

$U_{h}^{1}=P_{h}(0,1) \otimes Q_{h}^{1}(0,1)=\left\{\sum_{n=1}^{l} f_{n}(x) g_{n}(y) \mid f_{n} \in P_{h}(0,1), g_{n} \in Q_{h}^{1}(0,1), l \in \mathbf{N}\right\}$, and $U_{h}^{2}=P_{h}(0,1) \otimes Q_{h}^{2}(0,1)$.

The essential property of $U_{h}$ which will allow us to prove condition (P1) is the following: if $u=\left(u^{1}, u^{2}\right) \in U_{h}$, then $\partial_{y} u^{1}(x, \cdot)$ and $u^{2}(x, \cdot)$ are both in the same space $Q_{h}^{2}(0,1) \forall x \in(0,1)$. This property is used in the proof of Lemma 5.3.

Define furthermore the infinite-dimensional subspaces $W_{h}^{1}=H_{\#}^{1}(0,1) \otimes Q_{h}^{1}(0,1)$, $W_{h}^{2}=H_{\#}^{1}(0,1) \otimes Q_{h}^{2}(0,1)$ and $W_{h}=W_{h}^{1} \times W_{h}^{2}$. We have $U_{h} \subset W_{h} \subset U$.

4. Results. Let $p_{h}: U \rightarrow U_{h}$ be the $(\cdot, \cdot)_{V}$-projection.

THEOREM 4.1. There exists $\varepsilon_{h}>0$ with $\lim _{h \rightarrow 0} \varepsilon_{h}=0$ such that

$$
\left\|\left(I-p_{h}\right) T u\right\|_{V} \leqslant \varepsilon_{h}\|u\|_{V} \quad \forall u \in W_{h} .
$$

THEOREM 4.2. $\lim _{h \rightarrow 0}\left\|\left(I-p_{h}\right) u\right\|_{V}=0 \forall u \in U$.

Theorems 4.1 and 4.2 show that the properties (P1) and (P2) are simultaneously satisfied for the triples $\left(U,\left\{U_{h}\right\}_{0<h \leqslant 1}, T\right)$ and $\left(U,\left\{W_{h}\right\}_{0<h \leqslant 1}, T\right)$.

5. Proof. The density of $\mathscr{D}_{0} \times \mathscr{D}_{0}$ in $U$ and classical results of interpolation imply Theorem 4.2. Let us prove Theorem 4.1 in some detail.

For $n \in \mathbf{N}$, we denote by $H_{x}^{n}(\Omega)$ the completion of $\mathscr{Q}$ for the norm

$$
\|u\|_{n, x, \Omega}=\left(\sum_{i=0}^{n}\left\|\left(\partial_{x}\right)^{i} u\right\|_{0, \Omega}^{2}\right)^{1 / 2} \text {. }
$$

Let

$$
\begin{aligned}
H_{\#}^{n}(0,1)=\left\{f \in L^{2}(0,1) \mid f^{(i)} \in L^{2}(0,1) \forall 0 \leqslant i \leqslant n, f^{(i)}(0)\right. \\
\\
\left.=f^{(i)}(1) \forall 0 \leqslant i \leqslant n-1\right\}
\end{aligned}
$$

with the norm $\|f\|_{n,(0,1)}=\left(\sum_{i=0}^{n}\left\|f^{(i)}\right\|_{0,(0,1)}^{2}\right)^{1 / 2}\left(\|\cdot\|_{0,(0,1)}\right.$ is the norm in $\left.L^{2}(0,1)\right)$. We have that $U^{2}=V^{2}=H_{x}^{1}(\Omega)$.

Let $\alpha(\cdot, \cdot): H_{x}^{1}(\Omega) \times H_{x}^{1}(\Omega) \rightarrow \mathrm{C}$ and $\beta(\cdot, \cdot): L^{2}(\Omega) \times H_{x}^{1}(\Omega) \rightarrow \mathrm{C}$ be the two sesquilinear forms defined by

$$
\alpha(u, v)=\sum_{k, \tilde{k}=0}^{1} \int_{\Omega} \alpha_{k, \tilde{k}}\left(\partial_{x}\right)^{k} \overline{\left(\partial_{x}\right)^{k} v} \text { and } \beta(u, v)=\int_{\Omega} \beta \overline{\left(\partial_{x}\right)^{q} v}
$$


where $\alpha_{k, \tilde{k}}, \beta \in \mathscr{D}$ and $0 \leqslant q \leqslant 1$. Suppose that $\alpha(\cdot, \cdot)$ is coercive on $H_{x}^{1}(\Omega)$, and define $S: L^{2}(\Omega) \rightarrow H_{x}^{1}(\Omega)$ by $\alpha(S u, v)=\beta(u, v) \forall v \in H_{x}^{1}(\Omega), u \in L^{2}(\Omega)$. We remark that $S u \in H_{x}^{2}(\Omega) \forall u \in H_{x}^{q}(\Omega)$. This property can be proven with the same arguments as in Theorem 2.1.

Proposition 5.1. There exists $\varepsilon_{h}>0$ with $\lim _{h \rightarrow 0} \varepsilon_{h}=0$ such that $\forall u \in H_{\#}^{q}(0,1)$ $\otimes Q_{h}^{2}(0,1) \exists w_{h} \in U_{h}^{2}$ satisfying $\left\|S u-w_{h}\right\|_{1, x, \Omega} \leqslant \varepsilon_{h}\|u\|_{q, x, \Omega}$.

The proof of Proposition 5.1 is given at the end of the paper for the case where coefficients $\alpha_{k, \tilde{k}}$ and $\beta$ are independent of $y$.

Define the hermitian sesquilinear forms $a_{0}^{1}(\cdot, \cdot): U^{1} \times U^{1} \rightarrow \mathbf{C}, a_{0}^{2}(\cdot, \cdot): U^{2} \times$ $U^{2} \rightarrow \mathrm{C}$ and $a_{0}(\cdot, \cdot), \delta a(\cdot, \cdot): \quad U \times U \rightarrow \mathrm{C}$ by $a_{0}^{1}\left(u^{1}, v^{1}\right)=a\left(\left(u^{1}, 0\right),\left(v^{1}, 0\right)\right)$, $a_{0}^{2}\left(u^{2}, v^{2}\right)=a\left(\left(0, u^{2}\right),\left(0, v^{2}\right)\right), a_{0}(u, v)=a_{0}^{1}\left(u^{1}, v^{1}\right)+a_{0}^{2}\left(u^{2}, v^{2}\right)$ and $\delta a(u, v)=$ $a(u, v)-a_{0}(u, v) \forall u=\left(u^{1}, u^{2}\right), v=\left(v^{1}, v^{2}\right) \in U . a_{0}(\cdot, \cdot)\left(\operatorname{resp} . a_{0}^{1}(\cdot, \cdot), a_{0}^{2}(\cdot, \cdot)\right)$ is coercive on $U$ (resp. on $U^{1}$, on $U^{2}$ ).

Define the operators $T_{0}, R: U \rightarrow U$ by $a_{0}\left(T_{0} u, v\right)=b(u, v)$ and $a_{0}(R u, v)=$ $\delta a(u, v) \forall u, v \in U$.

LEMMA 5.2. There exists $\varepsilon_{h}>0$ with $\lim _{h \rightarrow 0} \varepsilon_{h}=0$ such that

$$
\left\|\left(I-p_{h}\right) T_{0} u\right\|_{V} \leqslant \varepsilon_{h}\|u\|_{V} \quad \forall u \in W_{h} .
$$

Proof. Suppose that $B_{22}=0$. In this case, $T_{0}$ is compact by compactness of the injection from $U^{1}$ into $L^{2}(\Omega)$. Thus, the lemma follows from Proposition 1.1 and Theorem 4.2.

By linearity, we can suppose now that $b(u, v)=\int_{\Omega} B_{22} u^{2} \bar{v}^{2}$. By Proposition 5.1 with $\alpha(\cdot, \cdot)=a_{0}^{2}(\cdot, \cdot), \beta=B_{22}$ and $q=0, T_{0} u=\left(0, S u^{2}\right)$ and $\forall v \in W_{h}^{2} \subset L^{2}(0,1)$ $\otimes Q_{h}^{2}(0,1), \exists w_{h} \in U_{h}^{2}$ such that $\left\|S v-w_{h}\right\|_{1, x, \Omega} \leqslant \varepsilon_{h}\|v\|_{0, \Omega}$. Consequently

$$
\left\|\left(I-p_{h}\right) T_{0} u\right\|_{V} \leqslant\left\|S u^{2}-w_{h}\right\|_{1, x, \Omega} \leqslant \varepsilon_{h}\|u\|_{V} .
$$

LEMMA 5.3. There exists $\varepsilon_{h}>0$ with $\lim _{h \rightarrow 0} \varepsilon_{h}=0$ such that $\forall u \in W_{h}$ satisfying $\left(\partial_{x}\right)^{i} u \in W_{h} \forall 0 \leqslant i \leqslant 2$. We have

$$
\left\|\left(I-p_{h}\right) R u\right\|_{V} \leqslant \varepsilon_{h} \sum_{i=0}^{2}\left\|\left(\partial_{x}\right)^{i} u\right\|_{V} .
$$

Proof. It suffices to prove Lemma 5.3 separately for each term of $\delta a(\cdot, \cdot)$. We shall do it for two of them. The proofs are similar for the other ones.

Consider first the case where $\delta a(u, v)=\int_{\Omega} A_{25} \partial_{x} u^{2} \partial_{x} v^{1}$.

We define the space $E=\left\{u \in U \mid\left(\partial_{x}\right)^{i} u \in U \forall 0 \leqslant i \leqslant 2\right\}$ with norm $\|u\|_{E}=$ $\sum_{i=1}^{2}\left\|\left(\partial_{x}\right)^{i} u\right\|_{V}$. We show that $R: E \rightarrow U$ is compact by the assumption of periodicity, $\delta a(u, v)=-\int_{\Omega} \partial_{x}\left(A_{25} \partial_{x} u^{2}\right) v^{-1}$. Since $\partial_{x}\left(A_{25} \partial_{x} u^{2}\right) \in L^{2}(\Omega) \forall u \in E, R: E \rightarrow U$ is compact by the compactness of the injection from $U^{1}$ into $L^{2}(\Omega)$. By Theorem 4.2 and Proposition 1.1, $\left\|\left(I-p_{h}\right) R u\right\|_{V} \leqslant \varepsilon_{h}\|u\|_{E} \forall u \in E$.

We treat next the term $\delta a(u, v)=\int_{\Omega} A_{35} \partial_{x} u^{2} \partial_{y} v^{1}$.

Let $u \in W_{h}$ be such that $\left(\partial_{x}\right)^{i} u \in W_{h} \forall 0 \leqslant i \leqslant 2$ and $w \in U^{1}$ be defined by $a_{0}^{1}(w, \varphi)=\delta a(u,(\varphi, 0)) \forall \varphi \in U^{1}$. In this proof, $\varepsilon_{h}$ is a generic positive function of $h$ such that $\lim _{h \rightarrow 0} \varepsilon_{h}=0$ and $c$ is a generic positive constant. Moreover $\varepsilon_{h}$ and $c$ are independent of $u$. Since $a_{0}^{1}(\cdot, \cdot)$ is hermitian and coercive on $U^{1}$, it is possible to show that $\exists \sigma>0$ such that $A_{33}(x, y) \geqslant \sigma \forall(x, y) \in \bar{\Omega}$. Set $z=A_{35} \partial_{x} u^{2} / A_{33}$. Then 
$z \in H_{x}^{2}(\Omega)$ and $\|z\|_{2, x, \Omega} \leqslant c\|u\|_{E}$. Using the arguments of the proof of Proposition 5.1 , it is possible to show the existence of $z_{h} \in U_{h}^{2}$ such that $\left\|z-z_{h}\right\|_{1, x, \Omega} \leqslant$ $\varepsilon_{h}\left\|\partial_{x} u^{2}\right\|_{2, x, \Omega} \leqslant \varepsilon_{h}\|u\|_{E}$.

Define $t, t_{h} \in V^{1}$ by $\partial_{y} t=z, \partial_{y} t_{h}=z_{h}, t(x, 0)=t_{h}(x, 0)=0 \forall x \in(0,1)$. Set $d(x, y)=y \cdot t(x, 1)$ and $d_{h}(x, y)=y \cdot t_{h}(x, 1), \tilde{t}=t-d$ and $\tilde{t}_{h}=t_{h}-d_{h}$. Then:

$$
\begin{gathered}
\|t\|_{2, x, \Omega}+\left\|\partial_{y} t\right\|_{2, x, \Omega} \leqslant c\|z\|_{2, x, \Omega} \leqslant c\|u\|_{E}, \\
\sum_{j=0}^{2}\left\|\left(\partial_{y}\right)^{j} d\right\|_{2, x, \Omega} \leqslant c\|t(\cdot, 1)\|_{2,(0,1)} \leqslant c\left(\|t\|_{2, x, \Omega}+\left\|\partial_{y} t\right\|_{2, x, \Omega}\right) \leqslant c\|u\|_{E},
\end{gathered}
$$

$$
\begin{gathered}
\left\|t-t_{h}\right\|_{1, x, \Omega}+\left\|\partial_{y}\left(t-t_{h}\right)\right\|_{1, x, \Omega} \leqslant c\left\|z-z_{h}\right\|_{1, x, \Omega} \leqslant \varepsilon_{h}\|u\|_{E}, \\
\left\|d-d_{h}\right\|_{1, \Omega} \leqslant c\left(\left\|t-t_{h}\right\|_{1, x, \Omega}+\left\|\partial_{y}\left(t-t_{h}\right)\right\|_{1, x, \Omega}\right) \leqslant \varepsilon_{h}\|u\|_{E}, \\
\left\|\tilde{t}-\tilde{t}_{h}\right\|_{1, \Omega} \leqslant \varepsilon_{h}\|u\|_{E},
\end{gathered}
$$

$\tilde{t}$ and $\tilde{t}_{h} \in V^{1}$, and, since $\tilde{t}(x, 0)=\tilde{t}_{h}(x, 0)=\tilde{t}(x, 1)=\tilde{t}_{h}(x, 1)=0, \forall x \in(0,1), \tilde{t}$ and $\tilde{t}_{h} \in U^{1}$.

Using the essential property of $U_{h}$ mentioned in Section 3, we prove that $\tilde{t}_{h} \in U_{h}^{1}$ : Set $z_{h}(x, y)=\sum_{n} f_{n}(x) g_{n}(y)$, where $f_{n} \in P_{h}(0,1)$ and $g_{n} \in Q_{h}^{2}(0,1)$. Let $\tilde{g}_{n}$ be defined by $d \tilde{g}_{n} / d y=g_{n}, \tilde{g}_{n}(0)=0$. We have $\tilde{t}_{h}(x, y)=\Sigma_{n} f_{n}(x) \varphi_{n}(y)$, where $\varphi_{n}(y)=$ $\tilde{g}_{n}(y)-y \tilde{g}_{n}(1)$. Since $\varphi_{n} \in Q_{h}^{1}(0,1), \tilde{t}_{h} \in P_{h}(0,1) \otimes Q_{h}^{1}(0,1)=U_{h}^{1}$. Define $w_{1}$ and $w_{2} \in W^{1}$ by $a_{0}^{1}\left(w_{1}, v\right)=\int_{\Omega} A_{33} \partial_{y} t \partial_{y} v-a_{0}^{1}(t, v) \forall v \in U^{1}$ and $a_{0}^{1}\left(w_{2}, v\right)=a_{0}^{1}(d, v)$ $\forall v \in U^{1}$.

We prove that the operator which associates $w_{1} \in U^{1}$ to $t \in\left\{\psi \in H_{x}^{2}(\Omega) \mid \partial_{y} \psi \in\right.$ $\left.H_{x}^{2}(\Omega)\right\}$ with the norm $\|\psi\|_{2, x, \Omega}+\left\|\partial_{y} \psi\right\|_{2, x, \Omega}$ is compact. We have

$$
\begin{aligned}
\int_{\Omega} A_{33} \partial_{y} t \overline{\partial_{y} v}- & a_{0}^{1}(t, v) \\
= & -\int_{\Omega} t\left(A_{11} \bar{v}+A_{21} \overline{\partial_{x} v}+A_{31} \overline{\partial_{y} v}\right) \\
& -\int_{\Omega} \partial_{x} t\left(A_{12} \bar{v}+A_{22} \overline{\partial_{x} v}+A_{32} \overline{\partial_{y} v}\right)-\int_{\Omega} A_{13} \partial_{y} t \bar{v}-\int_{\Omega} A_{23} \partial_{y} t \overline{\partial_{x} v}
\end{aligned}
$$

Since $t$ and $\partial_{x} t \in V^{1}$, the only term for which the compactness is not evident is $-\int_{\Omega} A_{23} \partial_{y} t \overline{\partial_{x} v}$. Let $\partial_{x}\left(A_{23} \partial_{y} t\right) \in L^{2}(\Omega)$ and $-\int_{\Omega} A_{23} \partial_{y} t \overline{\partial_{x} v}=\int_{\Omega} \partial_{x}\left(A_{23} \partial_{y} t\right) \bar{v}$. Thus the operator associating $w_{1}$ to $t$ is compact. Consequently, Theorem 4.2 and Proposition 1.1 imply the existence of $w_{1 h} \in U_{h}^{1}$ such that

$$
\left\|w_{1}-w_{1 h}\right\|_{1, \Omega} \leqslant \varepsilon_{h}\left(\|t\|_{2, x, \Omega}+\left\|\partial_{y} t\right\|_{2, x, \Omega}\right) \leqslant \varepsilon_{h}\|u\|_{E}
$$

In the same way, by the compactness of the injection from $\left\{\psi \in H_{x}^{2}(\Omega) \mid\left(\partial_{y}\right)^{j} \psi \in\right.$ $\left.H_{x}^{2}(\Omega) \forall 0 \leqslant j \leqslant 2\right\}$, with norm $\Sigma_{j=0}^{2}\left\|\left(\partial_{y}\right)^{j} \psi\right\|_{2, x, \Omega}$, into $V^{1}, \exists w_{2 h} \in U_{h}^{1}$ such that

$$
\left\|w_{2}-w_{2 h}\right\|_{1, \Omega} \leqslant \varepsilon_{h} \sum_{j=0}^{2}\left\|\left(\partial_{y}\right)^{j} d\right\|_{2, x, \Omega} \leqslant \varepsilon_{h}\|u\|_{E} .
$$


We prove that $w=\tilde{t}+w_{1}+w_{2}$. For $v \in U^{1}$, we have

$$
\begin{aligned}
a_{0}^{1}(w-\tilde{t}, v) & =\int_{\Omega} A_{35} \partial_{x} u^{2} \overline{\partial_{y} v}-a_{0}^{1}(t, v)+a_{0}^{1}(d, v) \\
& =\int_{\Omega} A_{33} \partial_{y} t \overline{\partial_{y} v}-a_{0}^{1}(t, v)+a_{0}^{1}(d, v)=a_{0}^{1}\left(w_{1}+w_{2}, v\right),
\end{aligned}
$$

i.e., $w=\tilde{t}+w_{1}+w_{2}$ since $t \in U^{1}$. Set $w_{h}=\tilde{t}_{h}+w_{1 h}+w_{2 h} \in U_{h}^{1}$.

By (1), (2), and (3), we finally obtain that

$$
\left\|\left(I-p_{h}\right) R u\right\|_{V} \leqslant\left\|w-w_{h}\right\|_{1, \Omega} \leqslant \varepsilon_{h}\|u\|_{E} .
$$

Define $\|u\|_{a_{0}}=\left(a_{0}(u, u)\right)^{1 / 2} \forall u \in U$, and set $\|R\|_{a_{0}}=\sup _{u \in U ;\|u\|_{a_{0}}=1}\|R u\|_{a_{0}}$.

LEMMA 5.4. $\|R\|_{a_{0}}<1$.

Proof. Since $R$ is selfadjoint, it is sufficient to prove that its spectrum $\sigma(R) \subset$ $(-1,1)$. We prove that the form $a_{\lambda}(u, v)=a_{0}(u, v)+\lambda \delta a(u, v)$ is coercive on $U$ $\forall \lambda \in[-1,1]$. By coerciveness of $a_{0}(\cdot, \cdot), \exists \sigma>0$ such that $\min \left(a(u, u), a_{0}(u, u)\right) \geqslant$ $\sigma\|u\|_{V}^{2} \forall u \in U$. Let $u \in U$ be fixed. If $\lambda \in[0,1]$,

$$
a_{\lambda}(u, u)=\lambda a(u, u)+(1-\lambda) a_{0}(u, u) \geqslant(\lambda \sigma+(1-\lambda) \sigma)\|u\|_{V}^{2}=\sigma\|u\|_{V}^{2} .
$$

If $\lambda \in[-1,0]$, set $v=\left(u^{1},-u^{2}\right)$ and so

$$
a_{\lambda}(u, u)=a_{0}(v, v)-\lambda \delta a(v, v)=a_{-\lambda}(v, v) \geqslant \sigma\|v\|_{V}^{2}=\sigma\|u\|_{V}^{2} .
$$

Consequently, if $\lambda \in[-1,1], \lambda \neq 0$, we have $a_{0}((I+\lambda R) u, v)=a_{\lambda}(u, v) \forall u, v \in U$ and, by coerciveness, $I+\lambda R: U \rightarrow U$ is an isomorphism. Letting $\lambda$ vary, we obtain that $\sigma(R) \subset(-1,1)$.

It is not difficult to prove that $W_{h}$ is a closed subspace in $U$. Define then $\Pi_{h}$ : $U \rightarrow U_{h}$ and $\hat{\Pi}_{h}: U \rightarrow W_{h}$ the $a_{0}(\cdot, \cdot)$-projections.

LEMMA 5.5. Let $n \in \mathbf{N}$ be fixed. If $u \in U$ satisfies the relation $\left(\partial_{x}\right)^{i} u \in U \forall 0 \leqslant i \leqslant$ $n$, then $\left(\partial_{x}\right)^{i} \hat{\Pi}_{h} u \in W_{h} \forall 0 \leqslant i \leqslant n$ and there exists $c>0$ independent of $u$ and of $h$ such that

$$
\sum_{i=0}^{n}\left\|\left(\partial_{x}\right)^{i} \hat{\Pi}_{h} u\right\|_{V} \leqslant c \sum_{i=0}^{n}\left\|\left(\partial_{x}\right)^{i} u\right\|_{V}
$$

This lemma can be proven with arguments similar to those in the proof of Theorem 2.1.

Proof of Theorem 4.1. Let $u \in W_{h}$. In this proof, $\varepsilon_{h}$ denotes a generic function of $h$ such that $\lim _{h \rightarrow 0} \varepsilon_{h}=0$ and $c$ represents a generic positive constant. Moreover, $\varepsilon_{h}$ and $c$ are independent of $u$. For $v \in U$, we have $a_{0}((I+R) T u, v)=a(T u, v)=$ $a_{0}\left(T_{0} u, v\right)$. Thus, $T u=T_{0} u-R T u$. Consequently, we have that

$$
\begin{aligned}
\left\|\left(I-\Pi_{h}\right) T u\right\|_{a_{0}} \leqslant & \left\|\left(I-\Pi_{h}\right) T_{0} u\right\|_{a_{0}}+\left\|\left(I-\Pi_{h}\right) R \hat{\Pi}_{h} T u\right\|_{a_{0}} \\
& +\left\|\left(I-\Pi_{h}\right) R\left(I-\hat{\Pi}_{h}\right) T u\right\|_{a_{0}} .
\end{aligned}
$$

By Lemma 5.2,

$$
\left\|\left(I-\Pi_{h}\right) T_{0} u\right\|_{a_{0}} \leqslant c\left\|\left(I-p_{h}\right) T_{0} u\right\|_{V} \leqslant \varepsilon_{h}\|u\|_{V} .
$$

By Theorem 2.1 and Lemmas 5.5 and 5.3,

$$
\left\|\left(I-\Pi_{h}\right) R \hat{\Pi}_{h} T u\right\|_{a_{0}} \leqslant c\left\|\left(I-p_{h}\right) R \hat{\Pi}_{h} T u\right\|_{V} \leqslant \varepsilon_{h} \sum_{i=0}^{2}\left\|\left(\partial_{x}\right)^{i} \hat{\Pi}_{h} T u\right\|_{V} \leqslant \varepsilon_{h}\|u\|_{V}
$$


Moreover,

$$
\left\|\left(I-\Pi_{h}\right) R\left(I-\hat{\Pi}_{h}\right) T u\right\|_{a_{0}} \leqslant\|R\|_{a_{0}}\left\|\left(I-\Pi_{h}\right) T u\right\|_{a_{0}} \leqslant\|R\|_{a_{0}}\left\|\left(I-\hat{\Pi}_{h}\right) T u\right\|_{a_{0}}
$$

since $U_{h} \subset W_{h}$. Consequently,

$$
\left\|\left(I-\Pi_{h}\right) T u\right\|_{a_{0}} \leqslant \varepsilon_{h}\|u\|_{V}+\|R\|_{a_{0}}\left\|\left(I-\Pi_{h}\right) T u\right\|_{a_{0}} .
$$

By Lemma 5.4, $\|R\|_{a_{0}}<1$ and consequently $\left\|\left(I-\Pi_{h}\right) T u\right\|_{a_{0}} \leqslant \varepsilon_{h}\|u\|_{V}$. Finally,

$$
\left\|\left(I-p_{h}\right) T u\right\|_{V} \leqslant c\left\|\left(I-\Pi_{h}\right) T u\right\|_{a_{0}} \leqslant \varepsilon_{h}\|u\|_{V} .
$$

We prove now Proposition 5.1. For the sake of simplicity we shall suppose that the coefficients $\alpha_{k \tilde{k}}$ and $\beta$ are independent of the variable $y$, and we give the proof in this case.

Define the sesquilinear forms $\stackrel{\alpha}{\alpha}(\cdot, \cdot): H_{\#}^{1}(0,1) \times H_{\#}^{1}(0,1) \rightarrow \mathrm{C}$ and $\stackrel{\circ}{\beta}(\cdot, \cdot)$ : $L^{2}(0,1) \times H_{\#}^{1}(0,1) \rightarrow \mathrm{C}$ by

$$
\stackrel{\alpha}{\alpha}(f, g)=\sum_{k, \tilde{k}=0}^{1} \int_{0}^{1} \alpha_{k, \tilde{k}}(x) f^{(k)} \overline{g^{(\tilde{k})}} d x \text { and } \dot{\beta}(f, g)=\int_{0}^{1} \beta(x) \overline{f g^{(q)}} d x \text {. }
$$

$\stackrel{\alpha}{\alpha}(\cdot, \cdot)$ is coercive on $H_{\#}^{1}(0,1)$. Let $\stackrel{\circ}{S}: L^{2}(0,1) \rightarrow H_{\#}^{1}(0,1)$ be defined by $\stackrel{\alpha}{\alpha}(\stackrel{\circ}{f}, g)=$ $\dot{\beta}(f, g) \forall g \in H_{\#}^{1}(0,1), f \in L^{2}(0,1)$.

Lemma 5.6. Let $u(x, y)=f(x) g(y)$, where $f$ and $g \in L^{2}(0,1)$. Then $\operatorname{Su}(x, y)=$ $(\stackrel{S}{f})(x) g(y)$.

Proof. One uses test functions in $H_{\#}^{1}(0,1) \otimes L^{2}(0,1)$ and the density of $H_{\#}^{1}(0,1) \otimes$ $L^{2}(0,1)$ in $H_{x}^{1}(0,1)$.

LemMa 5.7. There exists $\varepsilon_{h}>0$ with $\lim _{h \rightarrow 0} \varepsilon_{h}=0$ such that $\forall f \in H_{\#}^{q}(0,1), \exists g_{h} \in$ $P_{h}(0,1)$ satisfying $\left\|\stackrel{S}{S} f-g_{h}\right\|_{1,(0,1)} \leqslant \varepsilon_{h}\|f\|_{q,(0,1)}$.

Proof. By the compactness of the injection from $H_{\#}^{2}(0,1)$ into $H_{\#}^{1}(0,1)$, by density at the limit (property (P2)) of $\left\{P_{h}(0,1)\right\}_{0<h \leqslant 1}$ in $H_{\neq}^{1}(0,1)$, and by Proposition 1.1, there exists $\varepsilon_{h}>0$ with $\lim _{h \rightarrow 0} \varepsilon_{h}=0$ such that $\forall g \in H_{\#}^{2}(0,1)$, and there exists $g_{h} \in P_{h}(0,1)$ satisfying $\left\|g-g_{h}\right\|_{1,(0,1)} \leqslant \varepsilon_{h}\|g\|_{2,(0,1)}$. By elliptic regularity of $\dot{S}$, $\stackrel{\circ}{S} f \in H_{\#}^{2}(0,1) \quad \forall f \in H_{\#}^{q}(0,1)$ and there exists $c>0$ independent of $f$ such that $\|\stackrel{\circ}{S}\|_{2,(0,1)} \leqslant c\|f\|_{q,(0,1)}$. Setting $g=\stackrel{\circ}{S} f$, we obtain:

$$
\left\|\stackrel{S}{S}-g_{h}\right\|_{1,(0,1)} \leqslant \varepsilon_{h}\|\stackrel{\circ}{f}\|_{2,(0,1)} \leqslant \varepsilon_{h}\|f\|_{q,(0,1)} .
$$

Proof of Proposition 5.1. Let $\left\{g_{i} \mid 1 \leqslant i \leqslant r_{h}\right\}$ be a $(\cdot, \cdot)_{0,(0,1)}$-orthonormal basis of $Q_{h}^{2}(0,1) .\left(r_{h}=r N_{h}\right.$ is the dimension of $Q_{h}^{2}(0,1)$.) Let $u(x, y)=\sum_{i=1}^{r_{h}} f_{i}(x) g_{i}(y) \in$ $H_{\#}^{q}(0,1) \otimes Q_{h}^{2}(0,1)$. By Lemma 5.7, $\exists f_{i_{h}} \in P_{h}(0,1)$ such that $\left\|S f_{i}-f_{i_{h}}\right\|_{1,(0,1)} \leqslant$ $\varepsilon_{h}\left\|f_{i}\right\|_{q,(0,1)} \forall 1 \leqslant i \leqslant r_{h}$. Set $w_{h}(x, y)=\sum_{i=1}^{r_{h}} f_{i_{h}}(x) g_{i}(y) \in U_{h}^{2}$. By Lemma 5.6, we have

$$
\begin{aligned}
\left\|S u-w_{h}\right\|_{1, x, \Omega} & =\left(\sum_{i=1}^{r_{h}}\left\|\stackrel{\circ}{S} f_{i}-f_{i_{h}}\right\|_{1,(0,1)}^{2}\right)^{1 / 2} \\
& \leqslant \varepsilon_{h}\left(\sum_{i=1}^{r_{h}}\left\|f_{i}\right\|_{q,(0,1)}^{2}\right)^{1 / 2}=\varepsilon_{h}\|u\|_{q, x, \Omega}
\end{aligned}
$$


Département de Mathématiques

Ecole Polytechnique Fédérale de Lausanne (EPFL)

Avenue de Cour 61

1007 Lausanne, Switzerland

1. D. BERGER, Numerical Computations of the Ideal Magnetohydrodynamic Stability of Small Aspect Ratio Tokamaks, Thesis No 131, EPF-Lausanne, 1977.

2. J. Descloux, N. Nassif \& J. Rappaz, "On spectral approximation, Part 1: The problem of convergence, Part 2: Error estimates for the Galerkin method," RAIRO Numér. Anal., v. 12, No 2, 1978, pp. 97-119.

3. H. EvEquoz, Approximation Spectrale d'un Opérateur Lié à l'Étude de la Stabilité Magnétohydrodynamique d'un Plasma par une Méthode d'Éléments Finis Non Conforme, Thesis No 375, EPF-Lausanne, 1980.

4. G. FicherA, Linear Elliptic Differential Systems and Eigenvalue Problems, Lecture Notes in Math., vol. 8, Springer-Verlag, Berlin and New York, 1965.

5. R. GRUBER, Numerical Computations of the Magnetohydrodynamic Spectrum for One and Two Dimensional Equilibria Using Regular Finite Elements and Finite Hybrid Elements, Thesis No 246, EPF-Lausanne, 1976.

6. Y. JACCARD, Approximation Spectrale par la Méthode des Éléments Finis Conformes d'une Classe d'Opérateurs Non Compacts et Partiellement Réguliers, Thesis No 374, EPF-Lausanne, 1980.

7. N. Krall \& A. TrivelPiece, Principles of Plasma Physics, McGraw-Hill, New York, 1973.

8. J. RaPpaz, Approximation par la Méthode des Éléments Finis du Spectre d'un Opérateur Non Compact Donné par la Stabilité Magnétohydrodynamique d'un Plasma, Thesis No 239, EPF-Lausanne, 1976. 